\title{
Contenido de agua en suelos cultivados con Eucalyptus grandis y Pinus taeda. Análisis de un caso en la provincia de Entre Ríos, Argentina
}

\author{
Water content in soils cultivated with Eucalyptus grandis and Pinus taeda. \\ A case study in Entre Ríos province, Argentina
}

Eduardo Antonio Torrána, Juan Carlos Piter ${ }^{\mathrm{a} *}$

\author{
*Autor de correspondencia: aUniversidad Tecnológica Nacional, Facultad Regional Concepción del Uruguay, \\ Grupo de Estudio de Maderas (GEMA), Depto. de Ing. Civil, calle Ing. Pereira 676, (E3264BTD), Concepción del Uruguay,
} Entre Ríos, Argentina, tel./fax. 543442 425541, piterj@ frcu.utn.edu.ar

\begin{abstract}
SUMMARY
This paper reports the results of an investigation regarding the study of soil water content under a plantation of Eucalyptus grandis and another one of Pinus taeda located in a typical region for the cultivation of these species in Entre Ríos province, Argentina. Soil samples were monthly taken for 12 months up to a nominal depth of $2 \mathrm{~m}$ and soil water content was determined by means of the gravimetric method. Samples obtained from the E. grandis plantation exhibited higher water content than those taken from the $P$. taeda and a significant difference between the corresponding mean values was found. The statistical study also proved that the hypothesis of equal variances for the two groups of values may not be rejected and a detailed analysis showed similar variations when results were analysed as a function of the distance from the plantation border as well as the depth and the period of the year. Soil water content found under the two plantations was lower than the estimated withering point only in few spatially and temporally isolated opportunities.
\end{abstract}

Key words: soil water content, gravimetric method, forestry and environment.

\section{RESUMEN}

En el presente trabajo se presentan los resultados obtenidos de una investigación empírica orientada a estudiar el contenido de humedad del suelo bajo una plantación de Eucalyptus grandis y una de Pinus taeda ubicadas en una zona típica para el cultivo de estas especies en la provincia de Entre Ríos, Argentina. La extracción de suelo se efectuó hasta una profundidad nominal de 2 m, con una frecuencia mensual y durante 12 meses. La determinación del contenido de agua del suelo se llevó a cabo por medio del método gravimétrico. Los resultados mostraron un contenido de humedad mayor bajo la cobertura de E. grandis que bajo la de $P$. taeda. El análisis estadístico probó una diferencia significativa entre los valores medios de las muestras obtenidas bajo ambas especies y que la hipótesis de varianzas iguales en ellas no puede ser rechazada. Un estudio detallado puso en evidencia que la variación del contenido de agua del suelo fue similar bajo ambas especies tanto en función de la distancia a la frontera de las plantaciones como de la profundidad y de la época del año. La cantidad de agua que exhibió el suelo bajo ambas forestaciones se ubicó solo en pocas situaciones aisladas tanto temporal como espacialmente por debajo del punto de marchitez estimado para el mismo.

Palabras clave: humedad del suelo, método gravimétrico, forestaciones y ambiente.

\section{INTRODUCCIÓN}

En la provincia de Entre Ríos el género Eucalyptus ocupa el $90 \%$ de la superficie forestada, y está representado fundamentalmente por E. grandis Hill ex Maiden. Esta provincia es una de las más importantes productoras de esta especie en el país y en ella se encuentran también pequeñas cantidades de otras especies del mismo género, como E. globulus Labill. y E. dunnii Maiden. El 10\% restante de la superficie forestada está ocupada por especies del género Pinus, principalmente P. elliottii Engelm. y $P$. taeda L. Los crecimientos en los mejores suelos son comparables a los más altos a nivel internacional, situados entre 35 y $50 \mathrm{~m}^{3} \mathrm{ha}^{-1}$ año-1, y en algunos casos superando incluso los $50 \mathrm{~m}^{3} \mathrm{ha}^{-1} \mathrm{año}^{-1}$. Los turnos de corta usuales para el aserrado oscilan entre 10 y 15 años para Eucalyptus spp. y entre 20 y 22 años para Pinus spp. (INTA 1995).

El impacto producido sobre el ambiente por las forestaciones en general y por las del género Eucalyptus en particular, debido a los elevados requerimientos de agua $\mathrm{y}$ nutrientes, ha generado numerosas y variadas consideraciones (FAO 1987, Vihavainen 1995). En este sentido, y si bien todos los procesos ecológicos son importantes, el impacto sobre el agua que se encuentra en el suelo, específicamente aquella que las raíces de las plantas son 
capaces de utilizar, es uno de los aspectos que ha adquirido mayor importancia (Donoso 1992).

Tanto las forestaciones de $E$. grandis como la utilización de esta especie en calidad de cortina arbórea para proteger otras plantaciones, como es el caso de los cítricos de la provincia de Entre Ríos, Argentina, han originado opiniones encontradas e incertidumbre acerca de su relación con el agua del suelo en que se implantan. Independientemente de todos los factores que intervienen en un balance hídrico, la problemática planteada en este caso se focaliza en la existencia de agua en los suelos forestados, y en particular con E. grandis. En ese sentido los efectos que se le atribuyen a esta especie son contradictorios, ya que en algunos casos es señalada como causante de escasez de humedad en el suelo (Pérez Arrarte 2000, Carrere 2006), afectando el desarrollo de otros cultivos, y en otros esta posición es rechazada (Osava 2001, Rébori 2001, Díaz et al. 2006). La importancia de encontrar una respuesta a la interrogante planteada por medio de una investigación planificada se justifica con la destacada incidencia económica y social que este cultivo adquirió en la región, ya que el mismo se ha adaptado favorablemente y se desarrolla con gran velocidad de crecimiento.

Existen numerosas variables que intervienen en el proceso de interacción entre el suelo y las forestaciones, tales como el clima -incluyendo el régimen de precipitaciones- y el tipo de suelo, entre otras. Por tal razón, es conveniente encarar la solución al problema a través de un estudio realizado en el ambiente en el cual se insertan las plantaciones, para lo cual el estudio de caso resulta adecuado (Díaz et al. 2006). El conocimiento del contenido de humedad del suelo a distintas profundidades y distancias respecto de la frontera de la forestación, durante un período anual, en un suelo cubierto por una forestación de la especie más importante de la provincia, E. grandis, y del existente bajo otra especie considerada de referencia en la región, $P$. taeda, posibilita la realización de análisis comparativos que resultan de importancia para la toma de decisiones tanto en el sector gubernamental como en el productivo.

El objetivo de este trabajo es determinar el contenido de humedad del suelo en una forestación de E. grandis y en una de $P$. taeda, esta última considerada como referencia, y realizar un análisis comparativo de los resultados considerando su variación en función de la distancia a la frontera de las plantaciones, de la profundidad y de la época del año. Ambas forestaciones, de similar edad, son adyacentes en un mismo sitio característico de la región noreste de la provincia de Entre Ríos, Argentina.

\section{MÉTODOS}

Las forestaciones seleccionadas para la investigación se encuentran $7 \mathrm{~km}$ al sur de la localidad de Ubajay, a una latitud de $31^{\circ} 51^{\prime} \mathrm{S}$ y una longitud $58^{\circ} 20^{\prime} \mathrm{O}$. La de E. grandis, con 62 ha, tenía seis años de edad al comienzo del trabajo, una densidad de 800 árboles ha ${ }^{-1}$, una altura promedio de $15 \mathrm{~m}$ y un área basal de $28 \mathrm{~m}^{2}$ ha $^{-1}$, sin tratamientos silviculturales. La plantación de $P$. taeda, con 53 ha, contaba también con seis años de edad, una densidad de 1.000 árboles $\mathrm{ha}^{-1}$, una altura promedio de $8 \mathrm{~m}$ y un área basal de $18 \mathrm{~m}^{2} \mathrm{ha}^{-1}$, sin tratamientos silviculturales. Ambas plantaciones están adyacentes en un mismo terreno, el cual no presenta características en su superficie que pudiesen introducir variables no controladas con influencia sobre los resultados, tales como depresiones o canalizaciones. Durante el período de realización de las tareas de campo, el suelo ubicado debajo de ambas coberturas estuvo cubierto por una capa de hojarasca de aproximadamente $3 \mathrm{~cm}$ de espesor.

La topografía del área donde se encuentran las forestaciones presenta lomadas suaves con pendientes inferiores al $3 \%$. El suelo posee una textura arenosa típica de la unidad fisiográfica, "Terrazas arenosas onduladas a suavemente onduladas del Río Uruguay", comprendida en la Asociación Los Charrúas (INTA y Gobierno de Entre Ríos 2002). El clima se caracteriza por ser templado a cálido, húmedo y con veranos calurosos e inviernos no muy fríos. Los vientos predominantes son del Noroeste, Este y Sudeste, y les siguen en importancia los del Sur y el Norte, en tanto los del Oeste son muy escasos. Las heladas se producen entre mayo y septiembre y la humedad relativa media anual es del $75 \%$. Aunque las precipitaciones son más frecuentes en verano, la estación invernal es más húmeda que la estival (cuadro 1), debido al menor período de insolación y a la disminución de la intensidad de los rayos solares. El promedio mensual de precipitaciones para la zona,

Cuadro 1. Precipitaciones mensuales registradas durante el período de muestreo y medias para la región.

Monthly precipitations during the period of sampling and average for the region.

\begin{tabular}{|c|c|c|c|c|c|c|c|c|c|c|c|c|c|}
\hline Mes & Jun 06 & Jul 06 & Ago 06 & Set 06 & Oct 06 & Nov 06 & Dic 06 & Ene 07 & Feb 07 & Mar 07 & Abr 07 & May 07 & Jun 07 \\
\hline $\begin{array}{l}\text { Precipitaciones registradas } \\
(\mathrm{mm})^{(1)}\end{array}$ & 206 & 10 & 61 & 37 & 206 & 99 & 287 & 126 & 236 & 294 & 117 & 43 & 36 \\
\hline $\begin{array}{l}\text { Medias para la región } \\
(\mathrm{mm})^{(2)}\end{array}$ & 65 & 60 & 55 & 91 & 118 & 142 & 123 & 135 & 131 & 146 & 149 & 102 & 65 \\
\hline
\end{tabular}

(1): registradas por la empresa propietaria de las plantaciones; (2): para un período de 25 años (INTA 2001). 
calculado sobre un período de 25 años (INTA 2001), se presenta en el cuadro 1.

El registro de las precipitaciones correspondientes a cada mes del período de realización de los trabajos de campo estuvo a cargo de la empresa propietaria de las forestaciones, la cual realiza habitualmente esa tarea con un pluviómetro.

El perfil estratigráfico fue analizado en detalle a través de la realización de sendas calicatas en el interior de cada plantación, cuyas dimensiones nominales fueron $0,8 \mathrm{~m}$ de ancho, $1,5 \mathrm{~m}$ de largo y $2 \mathrm{~m}$ de profundidad. Éstas se ejecutaron en la cercanía de las zonas seleccionadas para la extracción de suelo, las que estuvieron separadas por una distancia de aproximadamente $40 \mathrm{~m}$. Las características del perfil fueron corroboradas también en cada sondeo utilizando para este fin una parte del suelo extraído para determinar su contenido de humedad. El lugar cultivado con $E$. grandis presentó, desde la superficie y hasta $0,72 \mathrm{~m}$ de profundidad, un suelo franco arenoso-limoso con bajo contenido de materia orgánica y de color castaño grisáceo. Posteriormente se encontró arena arcillosa hasta una profundidad de $0,87 \mathrm{~m}$, y luego, hasta $1,38 \mathrm{~m}$, arcilla arenosa rojizo-amarillenta, con motas rojas debido a la presencia de óxido de hierro. Desde ese nivel y hasta $1,5 \mathrm{~m}$ continuó con características similares pero sin una presencia tan marcada de óxido de hierro. Más abajo se presentó nuevamente el suelo franco arenoso-limoso hasta la aparición de un estrato de arenisca continua, muy compacta, con un nivel superior que oscilaba entre $1,8 \mathrm{~m}$ y $2 \mathrm{~m}$ de profundidad. Bajo la forestación de P. taeda se encontró un perfil similar al anterior, lo cual es coherente con la ubicación adyacente de ambas plantaciones. El manto superior de suelo franco arenoso-limoso se extendió en este caso hasta 0,6 $\mathrm{m}$ y luego el estrato de arena arcillosa hasta $0,97 \mathrm{~m}$. A partir de esta profundidad y hasta $1,1 \mathrm{~m}$ se encontró un estrato delgado de grava arcillosa compacta, posteriormente arena arcillosa, que en profundidad incrementó en forma gradual la proporción de arena hasta el fin de la excavación, que en este caso alcanzó los $2 \mathrm{~m}$ sin evidenciar la presencia del estrato de arenisca continua ya mencionado. La densidad del suelo seco, determinada por medio del método del cono de arena, respondió a valores típicos para el perfil encontrado, situándose en un rango de $1.400 \mathrm{~kg} \mathrm{~m}^{-3}$ a $1.760 \mathrm{~kg} \mathrm{~m}^{-3}$. La descripción de la textura del suelo se efectuó mediante sedimentación y utilizándose una cartilla granulométrica de comparación Soil Test. Se efectuó también un análisis microscópico utilizando un microscopio Olympus con aumentos correspondientes a oculares $10 \mathrm{x}$ y objetivos $2 \mathrm{x}$. El modelo utilizado permitió además realizar aumentos de 1 x a 4 x (zoom) sobre los anteriores. Para la clasificación edafológica se utilizó la carta de suelos realizada por el Instituto Nacional de Tecnología Agropecuaria para la región.

Con el objetivo de determinar el contenido de humedad del suelo, tanto de la frontera como del interior de cada forestación, se extrajo suelo de seis perforaciones ubicadas al azar en zonas donde no se observaron irregularidades locales. La zona colindante a la frontera de ambas plantaciones estaba cubierta por gramíneas, las que no superaban los $10 \mathrm{~cm}$ de altura. En consecuencia, su influencia sobre el contenido de humedad del suelo puede considerarse de muy escasa entidad considerando el objetivo planteado en este trabajo. La ubicación de las perforaciones, siguiendo el mismo criterio para ambas forestaciones, fue la siguiente: dos sobre la frontera, dos a $5 \mathrm{~m}$ de la misma hacia el interior y las otras dos a $10 \mathrm{~m}$ de ella también hacia el interior de la forestación. Teniendo en cuenta antecedentes que reportan para estos casos la presencia de la casi totalidad de las raíces entre la superficie y los $2 \mathrm{~m}$ (Lima 1996, Rébori 2001, Soares y Almeida 2001, Zhou et al. 2002, Lane et al. 2004, Radersma y Ong 2004), los sondeos se realizaron hasta una profundidad máxima de $2 \mathrm{~m}$, efectuándose cinco extracciones de suelo de cada uno, procedentes de la superficie y luego cada $0,5 \mathrm{~m}$ a partir de la misma. Se llevaron a cabo 12 extracciones entre junio de 2006 y junio de 2007, con una frecuencia mensual, con la excepción del mes de marzo de 2007 debido a que las condiciones meteorológicas y de acceso vehicular no lo permitieron. En cada oportunidad la toma de suelo se materializó durante el mismo día bajo ambas forestaciones. Luego de ubicadas las localizaciones programadas para los sondeos, los lugares precisos para efectuar las perforaciones se seleccionaron en todos los casos procurando que se encontrasen en puntos equidistantes a los árboles más próximos, con el fin de minimizar la probabilidad de que alguna raíz importante obstaculizara la penetración del equipo. Si bien en todas las oportunidades los pozos se rellenaron con el suelo remanente al finalizar las tareas, se adoptó el criterio de mantener una distancia mínima de $1 \mathrm{~m}$ entre un nuevo sondeo y otro ejecutado el mes anterior, con el fin de evitar posibles alteraciones en el entorno cercano de la perforación. Considerando que en cada plantación se planificaron seis sondeos, con extracción de suelo de cinco profundidades en 12 oportunidades durante el período ya mencionado, la cantidad de determinaciones programadas para el contenido de humedad del terreno ubicado debajo de cada especie ascendió a 360. Las extracciones se ejecutaron empleando cuatro cucharas sacamuestras cilíndricas, de $0,35 \mathrm{~m}$ de longitud, con las correspondientes barras de operación, las que fueron especialmente diseñadas para este trabajo. Una vez obtenido, el suelo fue colocado en bolsas de polietileno con doble envoltura y cerradas convenientemente para evitar la evaporación del agua contenida, las que fueron trasladadas al laboratorio inmediatamente luego de finalizar la tarea de campo.

La determinación inicial de la masa de cada extracción en el momento de acceder al laboratorio se efectuó utilizando una balanza Ohaus Explorer Pro, con capacidad máxima de $32 \mathrm{~kg}$ y una precisión de $0,1 \mathrm{~g}$. Estas determinaciones oscilaron, para el total de los casos, entre 300 g y 800 g, lo cual representó un tamaño suficiente para determinar el contenido de humedad del suelo y a 
su vez analizar las características de su composición. Con la finalidad de mantener la trazabilidad de los datos y facilitar los controles, toda la información fue registrada en una planilla preimpresa. Antes de fraccionar el material contenido en cada bolsa para determinar su contenido de agua se realizó un segundo pesaje con el fin de controlar eventuales variaciones de humedad entre el momento de la extracción y el procesamiento, ya que estas tareas no podían realizarse el mismo día. Los resultados confirmaron que las variaciones en el contenido de humedad del suelo en el período comprendido entre su extracción y su procesamiento final, el cual en ningún caso excedió una semana, fueron despreciables.

Para calcular el contenido de agua a través del método gravimétrico se determinó la masa húmeda $(m h)$ de una fracción de suelo proveniente de cada extracción. Con este fin se utilizó una balanza AND FX 3000 con una capacidad máxima de $3.100 \mathrm{~g}$ y una precisión de $0,01 \mathrm{~g}$. Las determinaciones efectuadas en esta etapa oscilaron en el rango comprendido entre 20 g y 60 g. Posteriormente el suelo se secó en una estufa marca Dalvo, Modelo BHRI, con regulación de temperatura. Ésta se configuró en $103^{\circ} \mathrm{C}$ y se mantuvo el material en ese régimen durante $24 \mathrm{~h}$ como mínimo. Cuando dos pesadas sucesivas con intervalo de $1 \mathrm{~h}$ indicaron una diferencia menor al $0,1 \%$ se consideró alcanzada una masa constante. Este valor fue registrado como masa seca o anhidra ( $\mathrm{ma}$ ). Con los datos obtenidos, aplicando la expresión [1], se obtuvo el contenido de humedad del suelo extraído $(h g)$ como porcentaje de masa de agua base suelo seco:

$$
h g(\%)=\frac{(m h-m a)}{(m a)} \times 100
$$

Los datos obtenidos fueron ordenados y procesados utilizando computadora y software adecuados para darles tratamiento estadístico. La media aritmética fue adoptada como medida de centralidad y el coeficiente de variación como medida de la dispersión. Para este último caso fueron utilizados también los valores mínimos y máximos. El resultado del análisis estadístico para comparar los valores medios de dos muestras se expresó a través del valor de la probabilidad asociada $(P)$ obtenido con el estadístico t y el número de grados de libertad. El cumplimiento de la hipótesis de igualdad entre las variancias de las muestras analizadas se verificó utilizando la prueba de Levene.

\section{RESULTADOS}

La distribución de las lluvias durante la realización de las tareas de campo fue irregular (cuadro 1), donde se destaca la importante diferencia entre los registros de junio de 2006 (206 mm) e igual mes de 2007 (36 mm). Hubo dos períodos de escasas precipitaciones, el primero comprendido entre julio y septiembre de 2006, en el cual las lluvias medidas en los meses mencionados fueron muy inferiores a las medias mensuales correspondientes. Este período fue interrumpido en octubre con un registro muy superior a la media mensual. El otro se presentó al final de las tareas de campo, entre mayo y junio de 2007. Por su parte, entre diciembre de 2006 y marzo de 2007 las precipitaciones fueron abundantes, $y$, con la excepción de enero de 2007, todos los meses registraron en ese período valores superiores a las medias mensuales correspondientes reportadas para la región (INTA 2001).

Los resultados para el contenido de humedad del suelo, considerando en forma conjunta el total de determinaciones (cuadro 2), revelaron diferencias altamente significativas entre los valores medios obtenidos bajo ambas plantaciones $(P=0,001)$. Tanto el valor mínimo como el medio y el máximo fueron superiores bajo la cobertura de $E$. grandis que bajo la de $P$. taeda. A su vez, el coeficiente de variación indicó una similar dispersión de valores bajo ambas forestaciones, la cual fue confirmada por los resultados del análisis estadístico, que mostraron que la hipótesis de varianzas iguales no puede ser rechazada $(P=0,889)$. La existencia de 340 determinaciones para la plantación de $E$. grandis se debe a que fue imposible efectuar una extracción de las planificadas a la profundidad de $1,5 \mathrm{~m}$ y 19 extracciones de las previstas a 2 m (ver también cuadro 4). Esto fue motivado porque el estrato de arenisca continua, ya mencionado anteriormente, presentó un nivel algo superior al habitual en coincidencia con esas perforaciones.

Cuadro 2. Contenido de humedad del suelo bajo las plantaciones.

Soil water content under the plantations.

\begin{tabular}{lcc}
\hline Plantación & E. grandis & P. taeda \\
\hline Mínimo (\%) & 2,5 & 1,3 \\
Media (\%) & $9,4^{* * *}$ & $8,4^{* * *}$ \\
Máximo (\%) & 29,0 & 23,8 \\
CV (\%) & 41 & 46 \\
$\mathrm{n}$ & 340 & 360 \\
\hline
\end{tabular}

$\mathrm{CV}$ : coeficiente de variación; $\mathrm{n}$ : cantidad de determinaciones. $* * *$ : medias difieren significativamente entre rodales $(P<0,001)$.

La discriminación de los resultados considerando las distintas distancias a la frontera de las plantaciones (cuadro 3) indica que con la excepción del valor máximo encontrado a la distancia de $10 \mathrm{~m}$, donde el suelo bajo la cobertura de $P$. taeda presentó una cantidad de agua levemente superior al ubicado bajo E. grandis, en el resto de los casos el terreno bajo esta última especie exhibió un mayor contenido de humedad. No obstante, el análisis estadístico confirmó que 
la diferencia entre los valores medios obtenidos bajo ambas especies en cada posición fue significativa solamente a la distancia de $5 \mathrm{~m}(P=0,011)$. Los valores del coeficiente de variación fueron muy similares en cada ubicación bajo las dos plantaciones y los resultados de la prueba de Levene confirmaron valores no significativos de probabilidad para las tres distancias analizadas, por lo cual la hipótesis de varianzas iguales bajo ambas especies en ninguno de los tres casos puede ser rechazada.

Bajo las dos forestaciones los valores medios del contenido gravimétrico de agua mostraron un leve crecimiento desde el interior hacia la frontera. Sin embargo, desde el punto de vista estadístico la diferencia entre las medias correspondientes a dos posiciones consecutivas bajo una misma plantación en ningún caso fue significativa.

Cuadro 3. Contenido de humedad del suelo para distintas distancias desde la frontera de las plantaciones.

Soil water content for different distances from the border of the plantations.

\begin{tabular}{lcccccc}
\hline Distancia: & $10 \mathrm{~m}$ (interior) & \multicolumn{2}{c}{$5 \mathrm{~m}$ (interior) } & \multicolumn{2}{c}{$0 \mathrm{~m}$ (frontera) } \\
\hline Plantación: & \multicolumn{1}{c}{$E$} & \multicolumn{1}{c}{$P$} & \multicolumn{1}{c}{$E$} & \multicolumn{1}{c}{$P$} & \multicolumn{1}{c}{$E$} & \multicolumn{1}{c}{$P$} \\
\hline Mínimo (\%) & 2,9 & 1,6 & 2,5 & 2,2 & 3,1 & 1,3 \\
Media (\%) & 8,9 & 8,2 & $9,5^{*}$ & $8,2^{*}$ & 9,8 & 8,7 \\
Máximo (\%) & 17,3 & 18,2 & 21,6 & 18,1 & 29,0 & 23,8 \\
CV (\%) & 40 & 44 & 39 & 43 & 43 & 50 \\
n & 111 & 120 & 113 & 120 & 116 & 120 \\
\hline
\end{tabular}

E: Eucalyptus grandis; P: Pinus taeda; CV: coeficiente de variación; $\mathrm{n}$ : cantidad de determinaciones. *: medias difieren significativamente entre rodales $(P=0,011)$.

La presentación de resultados separadamente para cada profundidad analizada (cuadro 4) permite apreciar que la mayor cantidad de agua en el suelo bajo la cobertura de $E$. grandis se manifestó también en casi todas las profundidades. El valor máximo fue mayor bajo esta especie con la única excepción del correspondiente a $1,5 \mathrm{~m}$, y lo mismo ocurrió con el valor mínimo con la excepción de $1 \mathrm{~m}$ y 1,5 m. La diferencia entre los valores medios fue altamente significativa en superficie y a $2 \mathrm{~m}(P<0,001)$, en tanto que fue significativa a $0,5 \mathrm{~m}(P=0,032)$ y a $1,5 \mathrm{~m}(P=0,021)$. Únicamente a la profundidad de $1 \mathrm{~m}$ se encontró un valor medio menor bajo la plantación de $E$. grandis que bajo la de $P$. taeda, y los resultados de la prueba estadística confirmaron que solamente para esa profundidad la diferencia entre las medias no fue significativa. La similitud encontrada para la dispersión de valores bajo ambas especies en todas las distintas distancias a la frontera, ya presentada anteriormente, se volvió a manifestar en este caso con la sola excepción de los resultados correspondientes a $0,5 \mathrm{~m}$ de profundidad. Los valores obtenidos para el coeficiente de variación bajo ambas plantaciones difirieron particularmente a esa distancia de la superficie y los resultados de la prueba de Levene confirmaron que únicamente para esa profundidad se debe rechazar la hipótesis de varianzas iguales $(P<0,001)$.

Bajo las dos forestaciones los valores medios del contenido gravimétrico de agua crecieron desde la superficie hasta $1 \mathrm{~m}$ y luego disminuyeron hasta la máxima profundidad analizada. Los resultados mostraron que las diferencias entre las medias correspondientes a dos profundidades consecutivas bajo una misma plantación fueron altamente significativas en todos los casos $(P<0,001)$ bajo las dos coberturas.

La discriminación de resultados en función del mes de extracción (cuadro 5) muestra mayores valores medios del contenido gravimétrico de agua bajo la cobertura de E. grandis que bajo la de $P$. taeda en 10 de los 12 meses en los cuales se efectuaron extracciones, correspondiendo las excepciones a julio de 2006 y junio de 2007. No obstante, los resultados de la prueba estadística indicaron que la diferencia entre las medias calculadas bajo ambas coberturas fue significativa solamente en el mes de abril de 2007 ( $P=0,048)$. Tanto los mayores como los menores valores del coeficiente de variación se registraron simultáneamente para ambas especies. Los mayores corres-

Cuadro 4. Contenido de humedad del suelo para distintas profundidades.

Soil water content for different depths.

\begin{tabular}{lccccccccccc}
\hline Profundidad: & \multicolumn{2}{c}{$0 \mathrm{~m}$} & \multicolumn{2}{c}{$0,5 \mathrm{~m}$} & \multicolumn{2}{c}{$1 \mathrm{~m}$} & \multicolumn{2}{c}{$1,5 \mathrm{~m}$} & \multicolumn{2}{c}{$2 \mathrm{~m}$} \\
\hline Plantación: & $\mathrm{E}$ & $\mathrm{P}$ & $\mathrm{E}$ & $\mathrm{P}$ & $\mathrm{E}$ & $\mathrm{P}$ & $\mathrm{E}$ & $\mathrm{P}$ & $\mathrm{E}$ & $\mathrm{P}$ \\
\hline Mínimo (\%) & 2,5 & 1,3 & 3,0 & 2,7 & 2,9 & 5,6 & 4,5 & 5,3 & 4,1 & 3,7 \\
Media (\%) & $6,9 * *$ & $5,4^{* *}$ & $8,5^{*}$ & $7,1^{*}$ & 12,8 & 13,6 & $10,1^{*}$ & $9,1^{*}$ & $8,4^{* *}$ & $6,8^{* *}$ \\
Máximo (\%) & 11,9 & 11,1 & 29,0 & 16,2 & 21,6 & 20,4 & 15,6 & 23,8 & 15,5 & 13,2 \\
CV (\%) & 32 & 38 & 57 & 37 & 31 & 23 & 23 & 30 & 28 & 36 \\
$\mathrm{n}$ & 72 & 72 & 72 & 72 & 72 & 72 & 71 & 72 & 53 & 72 \\
\hline
\end{tabular}

E: Eucalyptus grandis; P: Pinus taeda; CV: coeficiente de variación; n: cantidad de determinaciones. ${ }^{a}$ Medias difieren significativamente entre rodales (**: $P<0,01 ; *: P<0,05)$. 
Cuadro 5. Contenido de humedad del suelo para cada mes de muestreo.

Soil water content for each month of sampling.

\begin{tabular}{|c|c|c|c|c|c|c|c|c|c|c|c|c|c|c|c|c|c|c|c|c|c|c|c|c|}
\hline \multirow{2}{*}{$\frac{\text { Mes: }}{\text { Plantación: }}$} & \multicolumn{2}{|c|}{ Jun 06} & \multicolumn{2}{|c|}{ Jul 06} & \multicolumn{2}{|c|}{ Ago 06} & \multicolumn{2}{|c|}{ Set 06} & \multicolumn{2}{|c|}{ Oct 06} & \multicolumn{2}{|c|}{ Nov 06} & \multicolumn{2}{|c|}{ Dic 06} & \multicolumn{2}{|c|}{ Ene 07} & \multicolumn{2}{|c|}{ Feb 07} & \multicolumn{2}{|c|}{ Abr 07} & \multicolumn{2}{|c|}{ May 07} & \multicolumn{2}{|c|}{ Jun 07} \\
\hline & 2 & $P$ & $E$ & $P$ & $E$ & $P$ & $E$ & $P$ & $\Gamma$ & $P$ & & & $\Gamma$ & & & & & & & & & & 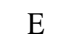 & $\mathrm{P}$ \\
\hline & & 4,4 & & & & & & & & 2,7 & & & & & & & & & & & & & & 12 \\
\hline & & & & & & & & & & 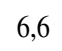 & & & & 10,3 & & & & 9 & & & &,$/$ & & 8,8 \\
\hline & & & & & & & & & & & & & & & & & & & & & & 6 & & 23, \\
\hline & & & & & & & & & & 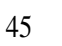 & & & 36 & & & & & & & & & & 41 & 37 \\
\hline & & & & & & & & & & 30 & & & 20 & & 20 & & & & & & 20 & & & 8 \\
\hline
\end{tabular}

E: Eucalyptus grandis; P: Pinus taeda; $\mathrm{CV}$ : coeficiente de variación; n: cantidad de determinaciones. *: medias difieren significativamente entre rodales $(P=0,048)$.

pondieron a septiembre de 2006, luego del primer período de escasas lluvias ya mencionado anteriormente. Los menores se registraron en febrero de 2007, en un período de abundantes precipitaciones (ver también el cuadro 1). Los resultados de la prueba estadística confirmaron que la hipótesis de varianzas iguales bajo ambas especies no puede ser rechazada, ya que en los 12 casos analizados se obtuvieron valores de probabilidad no significativos.

A su vez, la comparación de las cantidades de agua promedio del suelo con los correspondientes registros de precipitaciones (cuadro 1) evidencia también una relación entre estas dos variables durante los meses en que se realizaron las tareas de campo.

\section{DISCUSIÓN}

Los resultados presentados permiten conocer el contenido de humedad del suelo bajo las dos forestaciones, ubicadas adyacentemente sobre un mismo terreno. El análisis de los componentes de un balance hídrico que pudieron influir sobre los resultados, como la intercepción, requeriría considerar variables tales como el índice de área foliar y la intensidad de las precipitaciones. Esos estudios no están comprendidos en el objetivo del trabajo, pero los resultados obtenidos recibieron la influencia de tales componentes, $\mathrm{y}$ al respecto es necesario considerar que si bien ambas plantaciones tenían la misma edad, la de $P$. taeda poseía mayor densidad de árboles que la de E. grandis, pero en esta última especie los árboles tenían mayor altura y área basal (ver Métodos). No se conocen resultados de estudios sobre la intercepción en plantaciones de E. grandis cultivadas en la zona y con la edad de la seleccionada para este proyecto, pero se han publicado valores que oscilan entre $10,5 \%$ y $10,8 \%$ de las precipitaciones registradas en un año para forestaciones de la especie con edades comprendidas entre 9 y 10 años (Díaz et al. 2006). Se carece de datos al respecto para el $P$. taeda cultivado en la región, pero resultados de estudios llevados a cabo sobre una plantación con 10 años de edad en el sur de
Estados Unidos de Norteamérica (Hoover 1953) arrojaron una intercepción anual muy similar $(9,2 \%)$ a la expuesta anteriormente para la otra especie.

El mayor contenido de agua en el suelo cultivado con E. grandis que en el cultivado con P. taeda es confirmado para cada una de las posiciones respecto de la frontera en que se efectuó el análisis discriminado. El leve incremento de la cantidad de agua promedio desde el interior hacia la frontera de ambas plantaciones (cuadro 3) puede explicarse por una gradual disminución de la concentración de raíces hacia el exterior de ambas forestaciones (Radersma y Ong 2004).

El contenido de agua del suelo observado bajo ambas especies para las distintas profundidades estudiadas (cuadro 4), que crece desde la superficie hasta $1 \mathrm{~m}$ y luego disminuye hasta los $2 \mathrm{~m}$, presenta una variación congruente tanto con el perfil del suelo como con una mayor concentración de raíces en las cercanías de la superficie. La existencia de arcilla en el estrato encontrado a partir de $0,87 \mathrm{~m}$ bajo la cobertura de E. grandis y a partir de $0,97 \mathrm{~m}$ bajo la de P. taeda, como ya se describió anteriormente, constituye un factor que aumenta la impermeabilidad del suelo en ambos casos y en consecuencia la obtención del mayor contenido de agua a $1 \mathrm{~m}$ de profundidad es coherente con esta característica. Respecto de la densidad de raíces, Lima (1996) reporta la concentración del $98 \%$ del peso seco de las mismas entre la superficie y $0,6 \mathrm{~m}$ de profundidad para bosques de Eucalyptus spp. Patiño et al. (2004) informan que casi la totalidad de las raíces con funciones absorbentes del P. taeda también se concentra en los primeros $0,6 \mathrm{~m}$ del suelo, destacando que hasta los $0,4 \mathrm{~m}$ de profundidad se acumula el $85 \%$ de las mismas. Por su parte, Zhou et al. (2002) informan un mayor contenido de humedad en el suelo ubicado a profundidades comprendidas entre $0,6 \mathrm{~m}$ y $0,8 \mathrm{~m}$ para plantaciones de Eucalyptus spp.

La discriminación y el análisis de los resultados en función de la época del año (cuadro 5), además de confirmar una mayor cantidad de agua promedio en el terreno cultivado con $E$. grandis que en el ubicado bajo $P$. taeda en 10 de los 12 meses en los que se efectuaron 
extracciones, permite extender la comparación del contenido de humedad del suelo con su punto de marchitez a períodos con distinta cantidad de precipitaciones, lo cual tiene relación directa con la calidad de los cultivos que se producen (De Santa Olalla y De Juan Valero 1993). Para los suelos franco arenosos y franco areno-limosos predominantes en el perfil encontrado bajo ambas plantaciones puede considerarse que el punto de marchitez se ubica en un rango del contenido gravimétrico de humedad comprendido entre $4 \%$ y $9 \%$ (Dorronsoro 2008). La información de los cuadros 2 a 5 muestra que únicamente se encontraron valores mínimos inferiores a ese intervalo, ya que los valores medios y máximos superan el nivel inferior del rango en todos los casos. Un estudio detallado llevado a cabo con un mayor nivel de discriminación de los resultados que el exhibido en los cuadros precedentes, y que no se incluye en el presente por razones de espacio, muestra que solamente en ocasiones aisladas tanto en el espacio como en el tiempo el suelo cultivado con ambas especies presentó valores del contenido de humedad inferiores a su punto de marchitez estimado. En este sentido debe considerarse que durante la etapa de realización de las tareas de campo se registraron períodos en los cuales las precipitaciones registradas fueron muy inferiores a las medias mensuales correspondientes.

La determinación del contenido volumétrico de humedad, que permite conocer el volumen de suelo que está ocupado por agua, hubiese conducido en este caso a resultados similares a los obtenidos por el método gravimétrico cuando se comparan los valores encontrados bajo ambas coberturas. El contenido volumétrico de agua puede obtenerse multiplicando el contenido gravimétrico por la densidad aparente del suelo seco, y por lo tanto su cálculo requiere conocer la densidad de cada horizonte. En este caso, el perfil estratigráfico fue estudiado en detalle a través de la realización de sendas calicatas bajo ambas plantaciones y corroborado analizando las muestras en cada oportunidad en que se efectuaron extracciones de suelo, como se expresó anteriormente. El estudio confirmó características similares bajo las dos especies arbóreas ubicadas adyacentemente, incluyendo la densidad de los distintos estratos, cuyas pequeñas variaciones pueden considerarse normales dentro de un perfil de suelo típico para la región. En consecuencia, el estudio del contenido de humedad bajo ambas coberturas utilizando el contenido volumétrico hubiese diferido del expuesto anteriormente en que la comparación se habría efectuado entre los valores del contenido gravimétrico multiplicados por un valor prácticamente constante en cada caso, que es la densidad del suelo en el estrato analizado.

Los resultados obtenidos en esta investigación son congruentes con reportes previos (Osava 2001) que consideran que no existen fundamentos técnicos para afirmar que las forestaciones del género Eucalyptus causan una mayor disminución del contenido de agua del suelo en que se implantan que otras especies. Estos resultados alientan también la realización de nuevos estudios sobre la temática en zonas con otro perfil del suelo y distintas condiciones climáticas. A su vez, nuevas investigaciones podrían extender la determinación del contenido de agua del suelo hacia el exterior de las forestaciones.

\section{CONCLUSIONES}

El suelo bajo la cobertura de $E$. grandis presentó mayor contenido de agua que el ubicado bajo la plantación de $P$. taeda, y con una similar dispersión de valores.

El contenido de humedad promedio del suelo a distintas distancias a la frontera de las plantaciones fue en todos los casos mayor bajo el $E$. grandis que bajo el $P$. taeda y exhibió un leve crecimiento desde el interior hacia la frontera en ambas plantaciones.

El valor medio del contenido de humedad para distintas profundidades fue superior bajo el $E$. grandis que bajo el $P$. taeda con la excepción del encontrado a $1 \mathrm{~m}$. Bajo ambas plantaciones este valor se incrementó desde la superficie hasta $1 \mathrm{~m}$ y luego disminuyó hasta los $2 \mathrm{~m}$.

En 10 de los 12 meses en los cuales se efectuaron extracciones se encontró un mayor promedio del contenido de humedad bajo la cobertura de E. grandis que bajo la de $P$. taeda y una similar variación de esos valores bajo ambas especies.

El suelo bajo ambas plantaciones presentó en general una cantidad de agua superior a su punto de marchitez estimado, con la sola excepción de valores correspondientes a determinaciones que constituyeron casos aislados tanto en el espacio como en el tiempo.

\section{REFERENCIAS}

Carrere R. 2006. Maquillaje verde. Montevideo, Uruguay. Movimiento Mundial por los Bosques, Secretariado Internacional. $80 \mathrm{p}$.

De Santa Olalla MF, JA De Juan Valero. 1993. Agronomía del riego. Madrid, España. Mundi-Prensa. 731 p.

Díaz D, N Tesón, M García. 2006. Efectos ambientales de las forestaciones de eucaliptos en el noreste de Entre Ríos. Concordia, Argentina. XXI Jornadas Forestales de Entre Ríos. 15 p.

Donoso C. 1992. Ecología Forestal: el bosque y su medioambiente. Santiago, Chile. Editorial Universitaria. 368 p.

Dorronsoro C. 2008. Introducción a la edafología. Universidad de Granada. Consultado 28 mar. 2008. Disponible en http:// edafologia.ugr.es/introeda/tema00/progr.htm.

FAO (Organización de las Naciones Unidas para la Agricultura y la Alimentación, IT). 1987. Efectos ecológicos de los eucaliptos. 106 p. (Estudio FAO Montes 59).

Hoover MD. 1953. Interception of rainfall in a young loblolly pine plantation. U. S. Forest Serv., Southeast. Forest Exp. Sta. Paper 21, 13 p.

INTA (Instituto Nacional de Tecnología Agropecuaria, AR). 1995. Manual para productores de eucaliptos de la Mesopotamia Argentina. $162 \mathrm{p}$. 
INTA (Instituto Nacional de Tecnología Agropecuaria, AR). 2001. Novedades Forestales INTA Concordia $N^{\circ}$ 83. Consultado 27 feb. 2008. Disponible en http://www.mercoopsur.com. ar/forestales/notas/novedadesforestinta83.htm.

INTA (Instituto Nacional de Tecnología Agropecuaria, AR), Gobierno de Entre Ríos. 2002. Carta de suelos de la República Argentina. 276 p. (Serie de Relevamiento de Recursos Naturales $N^{\circ} 21$ ).

Lane PNJ, J Morris, Z Ningnan, Z Guangyi, A Guoyi, X Daping. 2004. Water balance of tropical eucalypt plantations in south-eastern China. Agricultural and Forest Meteorology 124: 253-267.

Lima WP. 1996. Impacto ambiental do eucalipto. São Paulo, Brasil. Editora da Universidade de São Paulo. 302 p.

Osava M (2001) Eucalipto contra viento y marea. Tierramérica. Consultado 26 feb. 2008. Disponible en http://www.tierramerica.net/2002/1021/articulo.shtml.

Patiño C, H Fassola, P Ferrere, R Pezzutti. 2004. Efectos de tratamientos silvícolas sobre la biomasa de raíces finas de Pinus taeda L. Eldorado, Argentina. XI Jornadas Técnicas Forestales y Ambientales de la Facultad de Ciencias Forestales UNAM y el INTA EEA Montecarlo. $11 \mathrm{p}$.
Pérez Arrarte C. 2000. Impacto de las plantaciones forestales en Uruguay. Biodiversidad 25: 7-15.

Radersma S, CK Ong. 2004. Spatial distribution of root length density and soil water of linear agroforestry system in subhumid Kenya: implications for agroforestry models. Forest Ecology and Management 188(1-3): 77-79.

Rébori M. 2001. Requerimientos de agua del Eucalyptus dunnii en su implantación y monte adulto. Medición y contribución al balance hídrico regional. In Investigación Forestal al Servicio de la Producción II. Buenos Aires, Argentina. SAGPyA. p. 207-236.

Soares JV, AC Almeida. 2001. Modelling the water balance and soil water fluxes in fast growing Eucalyptus plantation in Brazil. Journal of hydrology 253: 130-147.

Vihavainen T. 1995. Environmental aspects of timber. In Timber Engineering STEP 1. The Netherlands. Centrum Hout. p. A16/1-A16/8.

Zhou GY, JD Morris, JH Yan, Z Yu, SL Peng. 2002. Hydrological impacts of reafforestation with eucalypts and indigenous species: a case study in southern China. Forest Ecology and Management 167(11): 209-222.

Recibido: 07.11.08

Aceptado: 02.12.08 